Article

\title{
Improving the Signal to Noise Ratio of QTF Preamplifiers Dedicated for QEPAS Applications
}

\author{
Piotr Z. Wieczorek ${ }^{1}$, Tomasz Starecki ${ }^{1, *(D)}$ and Frank K. Tittel ${ }^{2}$ (D) \\ 1 Institute of Electronic Systems, Warsaw University of Technology, Nowowiejska 15/19, 00-665 Warsaw, \\ Poland; P.Z.Wieczorek@elka.pw.edu.pl \\ 2 Department of Electrical and Computer Engineering, Rice University, 6100 Main Street, Houston, \\ TX 77005, USA; fkt@rice.edu \\ * Correspondence: tomasz@starecki.com or t.starecki@elka.pw.edu.pl
}

Received: 27 May 2020; Accepted: 13 June 2020; Published: 15 June 2020

\begin{abstract}
The signal-to-noise ratio (SNR) is a major factor that limits the detection sensitivity of quartz-enhanced photoacoustic spectroscopy (QEPAS) sensors. The higher the electrical signal level compared to the noise amplitude is the lower the concentration of gases that can be detected. For this reason the preamplifier circuits used in QEPAS should be optimized for low-frequency narrow-band applications. Moreover, special care should be taken when choosing a particular operational amplifier in either a transimpedance or voltage (differential) configuration. It turns out that depending on the preamp topology different operational amplifier parameters should be carefully considered when a high SNR of the whole QEPAS system is required. In this article we analyzed the influence of the crucial parameters of low-noise operational preamplifiers used in QEPAS applications and show the resulting limitations of transimpedance and voltage configurations.
\end{abstract}

Keywords: quartz tuning fork; quartz enhanced photoacoustic; QEPAS sensor preamplifier; QTF front-end electronics

\section{Introduction}

Quartz enhanced photoacoustic spectroscopy (QEPAS) is a very sensitive spectroscopic technique in which detection of gas absorption induced with a laser is performed with a quartz tuning fork (QTF) [1-4]. Preamplifiers for piezoelectric sensors are implemented as voltage amplifiers or charge transimpedance amplifiers [5]. Until recently all QTF amplifiers dedicated for QEPAS were based on a transimpedance configuration, ut quartz has quite a high voltage constant $(118 \mathrm{~V} \cdot \mathrm{m} / \mathrm{N})$ and a low charge constant $(4.6 \mathrm{pC} / \mathrm{N})$, which results in good properties of the amplifiers based on the voltage configuration. It was demonstrated that properties of the preamplifier can be substantially improved if it is implemented in a voltage configuration. In particular it was shown that under identical stimulation a signal produced in the voltage amplifier configuration is over one order of magnitude higher than a signal from a transimpedance circuit [6]. In this paper we analyze the influence of the specifications of operational amplifiers on the properties of transimpedance and voltage configurations of QEPAS preamplifiers. The results show clearly that a voltage configuration is superior in terms of signal-to-noise ratio (SNR), which is an important conclusion taking into consideration that the QEPAS technique is commonly used in trace gas measurements.

\section{Experimental Setup}

Verification of the theoretical analysis of the QEPAS preamplifiers presented in [6] and experimental evaluation of the influence of noise characteristics of an op-amp (operational amplifier) on the properties of the preamplifier are based on measurements of the QTF preamplifiers implemented with 
several different op-amps tested in both configurations (voltage and transimpedance). The designed preamplifier was equipped with a socket (Figure 1), so that testing different models of the op-amp required only simple placing of the required model of the op-amp into the socket. For this reason all the tested op-amp models were in dual-in-line plastic (DIP) packages. Taking into consideration that the parasitic capacitance of the socket pins was less than $0.5 \mathrm{pF}$, while the input capacitance of the tested op-amps were at the level of a few $\mathrm{pF}$ and that the frequency of the circuit operation was low (tens of $\mathrm{kHz}$ ) influence of the socket on the circuit operation was negligible. A circuit diagram of the preamplifier circuit is given in Figure 2 (for the purpose of clarity of the power supply components visible in the photo from Figure 1 were omitted. However it should be mentioned that extreme care must be taken during the design of the preamplifier power supply. Selection of the preamplifier configuration (voltage or transimpedance) was possible by a simple change of the SW switch position. Due to such a solution, the op-amp and preamplifier configuration could be changed easily and without the need for preamplifier disassembly or readjustment of the measuring setup and allowed that the measurements could be performed in identical conditions. An additional ground copper layer below the PCB was used to reduce the possible influence of external E-M noise. For the tests the following op-amp models were chosen: OP27 (Analog Devices, Norwood, MA, USA), OPA227 (Texas Instruments, Dallas, TX, USA), TL071 (Texas Instruments, Dallas, TX, USA), OP42 (Analog Devices, Norwood, MA, USA) in order to evaluate influence of input voltage and current noise, input bias current, input impedance and op-amp bandwidth on the preamplifier properties. Selected parameters of these op-amp models are given in Table 1.

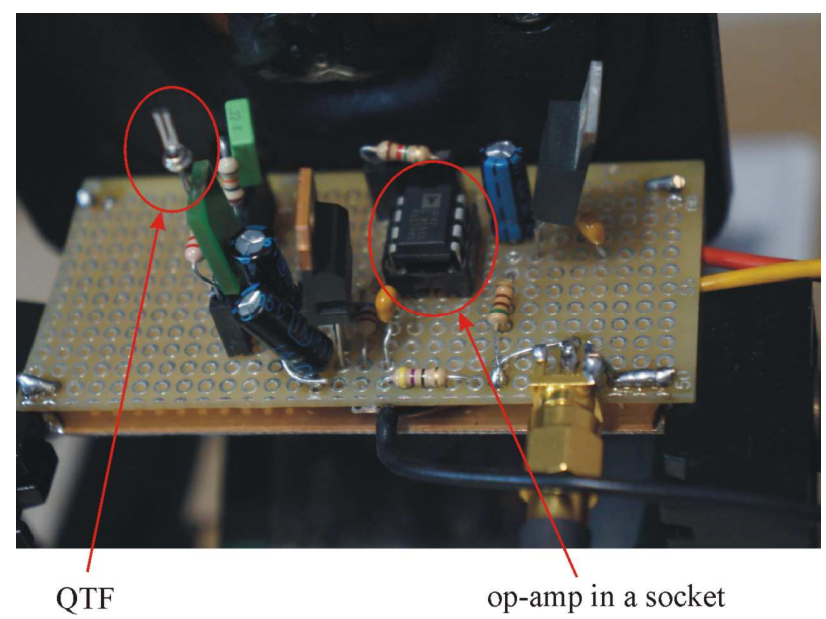

Figure 1. Photo of the preamplifier used in the measurements.

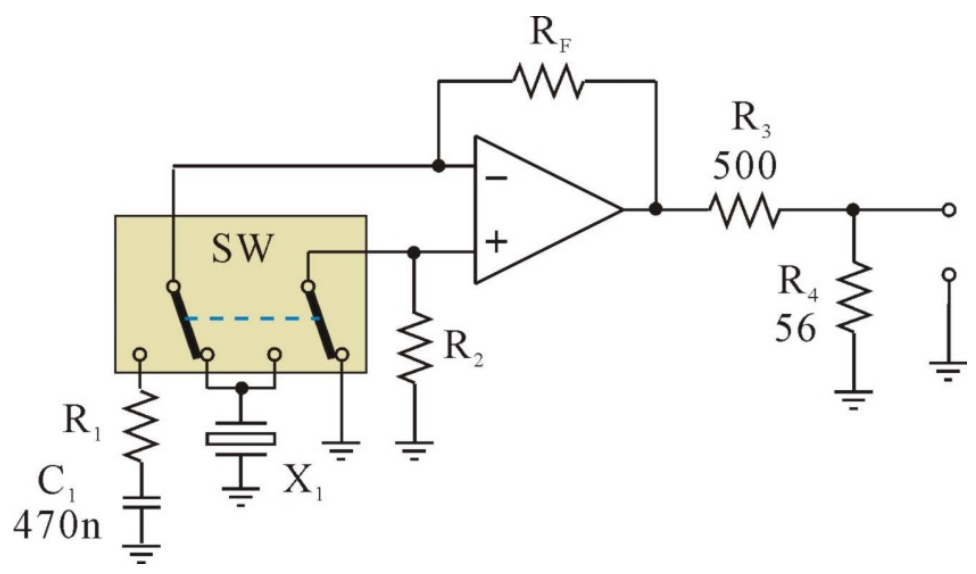

Figure 2. Circuit diagram of the tested preamplifier. 
Table 1. Selected parameters of the op-amps used in the measurements [7-10].

\begin{tabular}{|c|c|c|c|c|c|}
\hline $\begin{array}{l}\text { Op-Amp } \\
\text { Model }\end{array}$ & $\begin{array}{c}\text { Gain-Bandwidth } \\
\text { Product [MHz] }\end{array}$ & $\begin{array}{c}\text { Input Bias } \\
\text { Current [nA] }\end{array}$ & $\begin{array}{l}\text { Input Voltage Noise } \\
\left.\text { Density [nV/Hz }{ }^{1 / 2}\right]\end{array}$ & $\begin{array}{l}\text { Input Current Noise } \\
\text { Density }\left[\mathrm{pA} / \mathrm{Hz}^{1 / 2}\right]\end{array}$ & $\begin{array}{c}\text { Input Impedance } \\
\text { (Differential) [Ohm] }\end{array}$ \\
\hline OP27 & 8.0 & 15 & 3.0 & 0.4 & $4 \times 10^{6}$ \\
\hline OPA227 & 8.0 & 2.5 & 3.0 & 0.4 & $10^{7}$ \\
\hline TL071 & 3.0 & 0.065 & 18.0 & 0.01 & $10^{12}$ \\
\hline OP42 & 10.0 & 0.13 & 12.0 & 0.007 & $10^{12}$ \\
\hline
\end{tabular}

The circuit in Figure 2 can either act as a non-inverting amplifier, in which the voltage divider of the feedback loop is formed of $R F=1 M \Omega, R 1=10 \mathrm{k} \Omega$ and $C 1$ or a transimpedance amplifier, in which the $R F$ resistor defines the current-voltage conversion ratio. In the non-inverting configuration, the capacitor $\mathrm{C} 1$ acts as a low impedance device for relatively high frequencies. Therefore, when an AC (alternating current) voltage is applied at the circuit's input, the voltage gain is $k u=R 4(R F /(R 1)+1) /(R 3+R 4)$. However, when a DC voltage is applied the $\mathrm{C} 1$ capacitor acts as an infinite impedance and therefore the preamplifier acts as a voltage follower. Such a solution allowed us to minimize the output offset voltage resulting from the input bias currents causing voltage drops on R1 and RF resistors. Moreover, the undesirable low-frequency components are cut-off from the output signal. The whole circuit was subjected to further measurements performed with a spectrum analyzer (MDO3024 + MDO3SA, Tektronix, Beaverton, OR, USA), in which the default input resistance is $50 \Omega$ and the input signal is applied with a coaxial $50 \Omega$ cable. In order to limit the maximum output current of the amplifier resulting from the $50 \Omega$ load we used a voltage divider (current limiter) formed of resistors R3 and R4.

Every operation amplifier requires an input voltage (or current depending on the technology used) applied to inverting and non-inverting inputs. The QTF is a resonant circuit in which the DC resistance is infinite. Therefore a QTF cannot provide any bias voltage or current at the non-inverting input (in the non-inverting configuration provided by SW). For this reason the $\mathrm{R} 2$ resistor is present in the circuit in its non-inverting mode of operation. In our solution we have chosen $R 2=1 \mathrm{M} \Omega$. All the measurements were performed in a setup depicted in Figure 3. An DSOX 4104A oscilloscope (Keysight, Santa Rosa, CA, USA) used in the experiments has a built-in precise sine generator, whereas for the purpose of the precise noise and spectrum analysis a Tektronix MDO3024 was used, which allows performing high accuracy measurements with spectral averaging functionality.

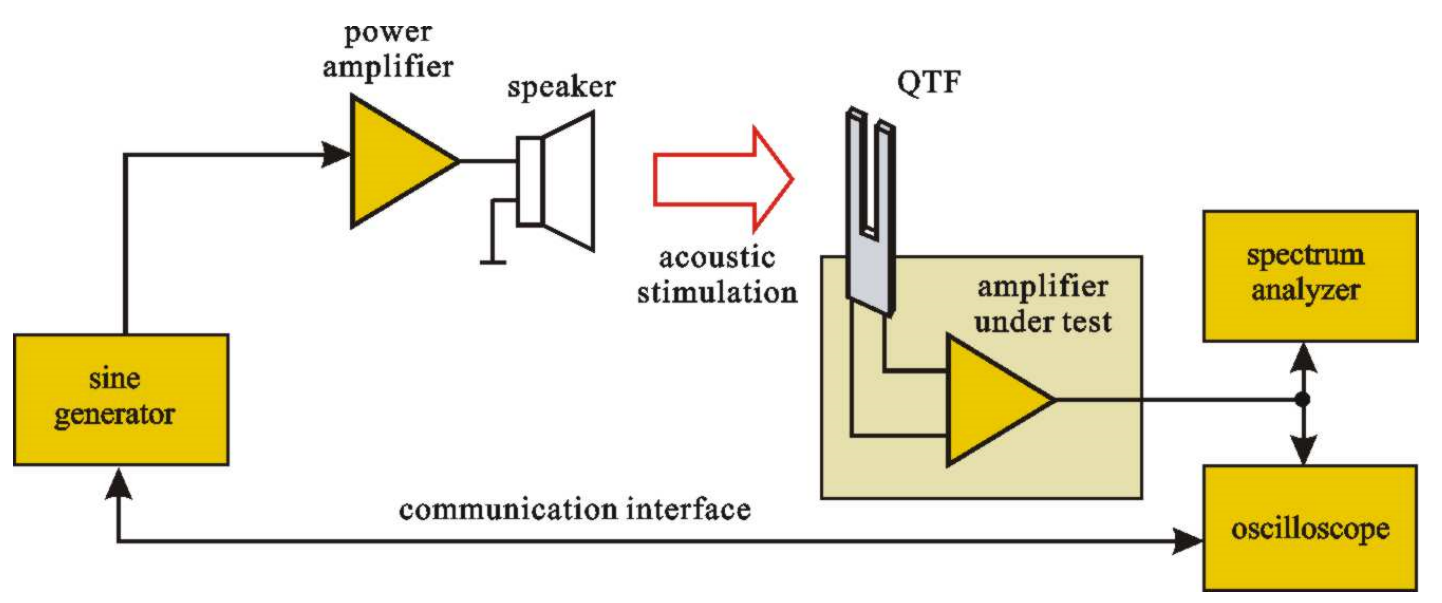

Figure 3. Setup used in the measurements.

It should be mentioned that the experimental setup in which the stimulation of the QTF was done with an acoustic signal produced with a speaker instead of a photoacoustic signal produced by a light beam passing between the QTF prongs and absorbed by the gas was used for this purpose. Due to such an approach the stimulation of the QTF was much stronger and making the measurements results virtually insensitive to external acoustic noise. Moreover, every replacement of the op-amp may slightly change the position of the QTF and if photoacoustic stimulation was used a realignment 
of the beam will be necessary. Unfortunately, every such readjustment might result in slightly different measuring conditions, while in the case of the purely acoustic stimulation produced with a speaker such realignments were not necessary and the measuring conditions can be considered as identical for all the investigated op-amps and their configurations. It was verified experimentally that under the mentioned acoustic stimulation of the tuning fork slight changes of the QTF position did not show any noticeable influence on the output signal level.

\section{Results and Discussion}

The preliminary measurements performed were aimed at evaluation of the selectivity of the sensor in both configurations (voltage and transimpedance). For this purpose the setup presented in Figure 3 was configured to measure the frequency response in a step by step mode, which means that every single point of the chart was obtained by setting the sine generator to a given frequency value and measuring the output signal from the amplifier with a lock-in technique and additional averaging. Due to use of such a method the obtained frequency response does not include any information about the signal-to-noise ratio, but expresses the output amplitude level from the QTF and selectivity of the sensor (ability of filtering the unwanted signals). The obtained results are presented in Figure 4. It is clearly visible that the output amplitude from the sensor working in a voltage mode is substantially higher, while selectivity of the sensor working in this mode is slightly worse than in a transimpedance configuration. It should be noticed, however, that in practical applications aimed at very sensitive measurements additional filtering and use of a lock-in (or another equivalent) technique are inevitable, no matter which of the configurations of the sensor is used.
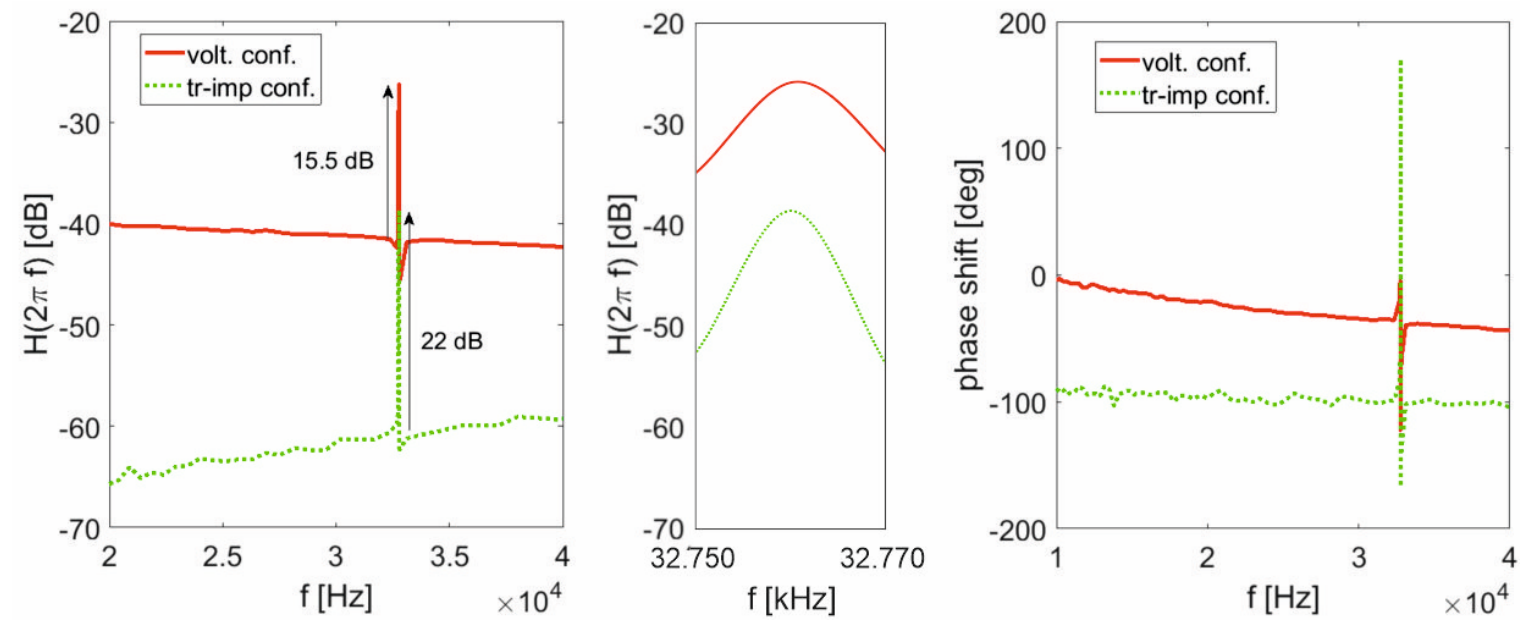

Figure 4. Frequency response of the QEPAS sensor with the preamplifier working in transimpedance and voltage configurations: amplitude (left), amplitude zoomed around the resonance frequency (middle) and phase (right) measured with the frequency resolution better than $0.1 \mathrm{~Hz}$.

The next set of the measurements was carried out with all the averaging capabilities switched off, in order to observe the signal-to-noise properties of the investigated circuits. At first, with the acoustical stimulation switched on, we used a spectrum analyzer, which performed a frequency sweep with narrowband measurements of the amplitude of the preamplifier output signal. All four op-amps working in both configurations were investigated in this manner and results of these measurements are presented in a form of curves in Figure 5. The SNR numbers given in Figure 5 were obtained separately. In this case at first the frequency of the acoustic stimulation was tuned to the QTF resonance (frequency at which the preamplifier output signal was reaching its maximum level) - the frequency was adjusted with the resolution better than $0.1 \mathrm{~Hz}$. The output signal level from the preamplifier was then measured (signal measurement). Then the acoustic stimulation was switched off and the output signal level from the preamplifier was measured again (noise measurement). Finally the two values 
were used to calculate the SNR. Such an approach was used, because if we deal with a narrowband operation, SNR is defined as a ratio of the signal level to the noise floor level (background noise level at the signal frequency). Taking into consideration that QEPAS signal is usually measured with the use of lock-in amplifiers (which limits the bandwidth to a fraction of $\mathrm{Hz}$ ), it is definitely a narrowband operation, and that is why the above definition applies. Results of these measurements are presented in Figure 5 and in Table 2.
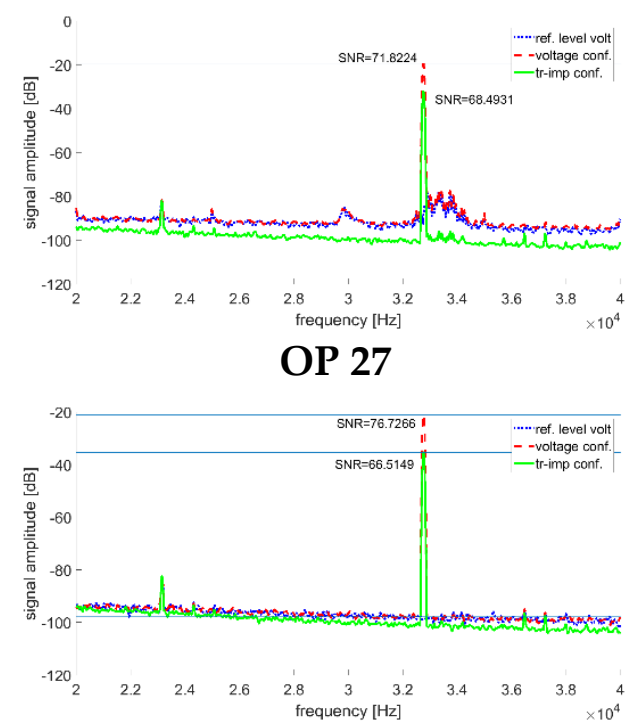

TL 071

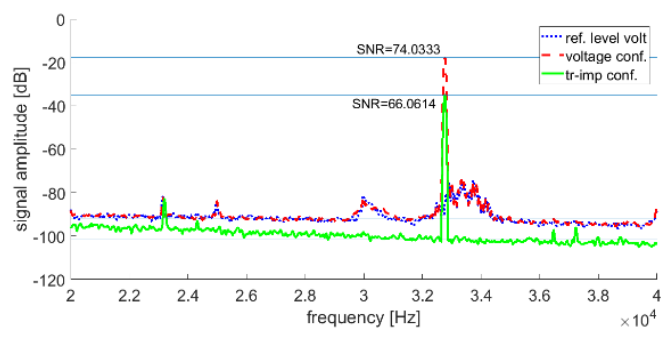

OP 227

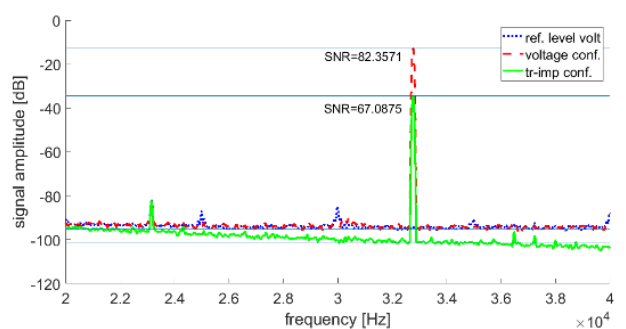

OP 42

Figure 5. Comparison of the signal level and noise properties of the QTF preamplifier with different operational amplifiers working in voltage and transimpedance modes.

Table 2. Measured amplitude of the signal from the sensor and signal-to-noise ratio for the QTF preamplifiers implemented with different operational amplifier models working in transimpedance and voltage configurations.

\begin{tabular}{ccccc}
\hline \multirow{2}{*}{ Op-Amp Model } & \multicolumn{2}{c}{ Output Amplitude [dB] } & \multicolumn{2}{c}{ SNR [dB] } \\
\cline { 2 - 5 } & Voltage & Transimpedance & Voltage & Transimpedance \\
\hline OP27 & -19.66 & -32.23 & 71.82 & 68.49 \\
OPA227 & -17.81 & -35.06 & 74.03 & 66.06 \\
TL071 & -21.01 & -35.35 & 76.73 & 66.51 \\
OP42 & -12.82 & -34.42 & 82.36 & 67.09 \\
\hline
\end{tabular}

The obtained results clearly show that in the case of all tested operational amplifier models the use of the QTF preamplifier operating in the voltage configuration gives better results in terms of both the output signal amplitude and signal-to-noise ratio. A comparison of the contents of Tables 1 and 2 leads to a conclusion that in the case of the voltage configuration the main factor which determines the noise properties is the current noise and definitely not the voltage noise. Such a result was predictable taking into consideration that the QTF has a relatively high impedance. For the same reason a too low input differential impedance may also reduce the signal-to-noise ratio of the voltage configuration. This is exactly the case of OP27 and OPA227, which have virtually identical voltage and current noise coefficients, but OP27 has a noticeably lower input impedance which is comparable with the QTF impedance. The TL071 amplifier shows worse results than OP42, because of a slightly higher voltage noise and a much lower gain-bandwidth product. Taking into consideration the gain of the voltage configuration, the upper corner frequency of the TL071 based voltage preamplifier is around $29 \mathrm{kHz}$, which is already below the resonance frequency of the QTF. Therefore the output amplitude for the TL071 amplifier is lower compared to other amplifiers taken into consideration in the non-inverting 
configuration. Nevertheless, due to a 40 times lower noise current compared to OP27 and OPA227 the overall SNR performance of TL071 in the non-inverting configuration is better. One can see that the highest voltage noise of TL071 compared to other considered operational amplifiers makes it the worst solution for a transimpedance configuration.

In order to check if the measurement results conform with our previous theoretical analysis presented in [6] we calculated preamplifier noise from the noise models discussed there. Certainly, it was not possible to calculate the absolute value of the preamplifier signal amplitude neither its SNR, as both these values are strongly influenced by many other factors. But assuming that the given noise models are correct, the ratio of the SNRs calculated and measured for two different op-amps working in the same configuration should be similar. To make the comparison easier we scaled up the calculated SNR values in such a way that the theoretical and measured SNRs of OP42 based preamplifier are identical. Theoretical SNRs of the remaining op-amps were scaled up accordingly. Comparison of such calculated and measured SNRs is given in Table 3.

Table 3. Comparison of theoretical and measured SNRs for preamplifiers implemented with different operational amplifier models working in transimpedance and voltage configurations.

\begin{tabular}{ccccc}
\hline \multirow{2}{*}{ Op-Amp Model } & \multicolumn{2}{c}{ Calculated SNR [dB] } & \multicolumn{2}{c}{ Measured SNR [dB] } \\
\cline { 2 - 5 } & Voltage & Transimpedance & Voltage & Transimpedance \\
\hline OP27 & 76.31 & 62.58 & 71.82 & 68.49 \\
OPA227 & 76.31 & 62.58 & 74.03 & 66.06 \\
TL071 & 78.75 & 66.43 & 76.73 & 66.51 \\
OP42 & 82.36 & 67.09 & 82.36 & 67.09 \\
\hline
\end{tabular}

Although there are some differences between the theoretical evaluation and the measurements, which may result from imperfect noise models of the preamplifiers, technological tolerance of the properties used in the theoretical estimations, etc., it is quite clear that all the previous conclusions stand valid.

\section{Conclusions}

The experimentally acquired data proved that a voltage configuration preamplifier has superior performance in comparison to the most commonly used transimpedance configuration preamplifiers. When selecting an operational amplifier dedicated for voltage mode QEPAS preamplifier, the choice should be based primarily on a low current noise coefficient op-amp model. The input voltage noise is less critical and certainly the lower, the better. Finally, if the gain of the voltage preamplifier is set to about 100 as in the investigated test circuit, the op-amp gain-bandwidth product should not be less than $8-10 \mathrm{MHz}$.

Author Contributions: Conceptualization, P.Z.W. and T.S.; Funding acquisition, F.K.T.; Investigation, P.Z.W.; Methodology, P.Z.W. and T.S.; Visualization, P.Z.W.; Writing —original draft, P.Z.W., T.S. and F.K.T.; Writing—review \& editing, P.Z.W., T.S. and F.K.T. All authors have read and agreed to the published version of the manuscript.

Funding: The work was supported from the statutory funds granted by the Polish Ministry of Science and Higher Education. Frank K. Tittel acknowledges the financial support a grant C-0586 from the Welch Foundation.

Conflicts of Interest: The authors declare no conflict of interest.

\section{References}

1. Patimisco, P.; Scamarcio, G.; Tittel, F.K.; Spagnolo, V. Quartz-enhanced photoacoustic spectroscopy: A review. Sensors 2014, 14, 6165-6206. [CrossRef] [PubMed]

2. Kosterev, A.A.; Bakhirkin, Y.A.; Curl, R.F.; Tittel, F.K. Quartz-enhanced photoacoustic spectroscopy. Opt. Lett. 2002, 27, 1902-1904. [CrossRef] [PubMed] 
3. Kosterev, A.A.; Tittel, F.K.; Serebryakov, D.V.; Malinovsky, A.L.; Morozov, I.V. Applications of quartz tuning forks in spectroscopic gas sensing. Rev. Sci. Instrum. 2005, 76, 043105. [CrossRef]

4. Kosterev, A.A.; Dong, L.; Thomazy, D.; Tittel, F.K.; Overby, S. QEPAS for chemical analysis of multi-component gas mixtures. Appl. Phys. B 2010, 101, 649-659. [CrossRef]

5. Starecki, T. Analog front-end circuitry in piezoelectric and microphone detection of photoacoustic signals. Int. J. Thermophys. 2014, 35, 2124-2139. [CrossRef]

6. Starecki, T.; Wieczorek, P.Z. A high sensitivity preamplifier for quartz tuning forks in QEPAS (Quartz Enhanced PhotoAcoustic Spectroscopy) applications. Sensors 2017, 17, 2528. [CrossRef] [PubMed]

7. Analog Devices, Low Noise, Precision Operational Amplifier OP27. Available online: https://www.analog. com/media/en/technical-documentation/data-sheets/OP27.pdf (accessed on 30 March 2019).

8. Texas Instruments, OPAx22x High Precision, Low Noise Operational Amplifiers. Available online: http: //www.ti.com/lit/ds/symlink/opa2228.pdf (accessed on 30 March 2019).

9. Texas Instruments, TL07xx Low-Noise JFET-Input Operational Amplifiers. Available online: http://www.ti. com/lit/ds/symlink/t1072.pdf (accessed on 30 March 2019).

10. Analog Devices, High Speed, Fast Settling, Precision Operational Amplifier OP42. Available online: https: //www.analog.com/media/en/technical-documentation/data-sheets/OP42.pdf (accessed on 30 March 2019).

(C) 2020 by the authors. Licensee MDPI, Basel, Switzerland. This article is an open access article distributed under the terms and conditions of the Creative Commons Attribution (CC BY) license (http://creativecommons.org/licenses/by/4.0/). 